\title{
Extended Anatomical Grading in Diffuse Axonal Injury Using MRI: Hemorrhagic Lesions in the Substantia Nigra and Mesencephalic Tegmentum Indicate Poor Long-Term Outcome
}

\author{
Sami Abu Hamdeh, Niklas Marklund, ${ }^{1}$ Marianne Lannsjö,2,3 Tim Howells, \\ Raili Raininko, Johan Wikström, and Per Enblad ${ }^{4}$
}

\begin{abstract}
Clinical outcome after traumatic diffuse axonal injury (DAI) is difficult to predict. In this study, three magnetic resonance imaging (MRI) sequences were used to quantify the anatomical distribution of lesions, to grade DAI according to the Adams grading system, and to evaluate the value of lesion localization in combination with clinical prognostic factors to improve outcome prediction. Thirty patients (mean 31.2 years \pm 14.3 standard deviation) with severe DAI (Glasgow Motor Score $[\mathrm{GMS}]<6$ ) examined with MRI within 1 week post-injury were included. Diffusion-weighted (DW), T2*-weighted gradient echo and susceptibility-weighted (SWI) sequences were used. Extended Glasgow outcome score was assessed after 6 months. Number of DW lesions in the thalamus, basal ganglia, and internal capsule and number of SWI lesions in the mesencephalon correlated significantly with outcome in univariate analysis. Age, GMS at admission, GMS at discharge, and low proportion of good monitoring time with cerebral perfusion pressure $<60 \mathrm{~mm} \mathrm{Hg}$ correlated significantly with outcome in univariate analysis. Multivariate analysis revealed an independent relation with poor outcome for age $(p=0.005)$ and lesions in the mesencephalic region corresponding to substantia nigra and tegmentum on SWI $(p=0.008)$. We conclude that higher age and lesions in substantia nigra and mesencephalic tegmentum indicate poor long-term outcome in DAI. We propose an extended MRI classification system based on four stages (stage I—hemispheric lesions, stage II — corpus callosum lesions, stage III—brainstem lesions, and stage IV—substantia nigra or mesencephalic tegmentum lesions); all are subdivided by age $(\geq /<30$ years).
\end{abstract}

Keywords: adult brain injury; axonal injury; head trauma; MRI; susceptibility weighted imaging

\section{Introduction}

$\mathbf{T}$ RAUMATIC BRAIN INJURY (TBI) remains a major cause of death and disability. TBI accounts for one-third of all injuryassociated deaths in the United States affecting all ages, ethnicities, and income groups. ${ }^{1}$ Traditionally, TBI may be divided by biomechanical and neuroradiological characteristics into focal (fracture, contusion, subdural or epidural hematoma) and diffuse injuries (diffuse axonal injury [DAI] and diffuse brain edema).

DAI is a common finding in patients with severe $\mathrm{TBI}^{2,3}$ and appears to be a more important predictor of poor neurological outcome and long-term cognitive and behavioral impairments than focal TBI. ${ }^{4-6}$ DAI is caused by angular or rotational acceleration-deceleration forces to the head resulting in stretching of brain tissue.

The histopathological grading of DAI, proposed by Adams and associates ${ }^{7}$ into grades 1-3 is based on the presence of axonal injury in the cerebral hemispheres with a predilection for the grey-white interface (grade 1), the corpus callosum (grade 2), and the dorsolateral, rostral brainstem (grade 3). Diffuse brain injuries can also be classified by the widely used Marshall computed tomography (CT) classification of TBI based on the

Departments of ${ }^{1}$ Neuroscience, Neurosurgery, ${ }^{2}$ Neuroscience, Rehabilitation Medicine, Uppsala University, Uppsala, Sweden.

${ }^{3}$ Center of Research and Development, Uppsala University/County Council of Gävleborg, Gävle Hospital, Gävle, Sweden.

${ }^{4}$ Department of Radiology, Uppsala University, Uppsala, Sweden.

(c) Sami Abu Hamdeh, et al., 2016; Published by Mary Ann Liebert, Inc. This Open Access article is distributed under the terms of the Creative Commons Attribution Noncommercial License (http://creativecommons.org/licenses/by-nc/4.0/), which permits any noncommercial use, distribution, and reproduction in any medium, provided the original author(s) and the source are credited. 
degree of compression of the mesencephalic cisterns, the degree of midline shift, and the presence or absence of one or more surgical mass lesions. ${ }^{8}$

The CT findings in DAI are typically limited to microhemorrhages in the white matter and/or traumatic edema of the brain. ${ }^{2,7,9}$ Magnetic resonance imaging (MRI) is a more sensitive method than $\mathrm{CT}$ to detect DAI and capable of visualizing even microscopic amounts of blood products. ${ }^{10}$ MRI sequences sensitive for hemorrhage such as $\mathrm{T} 2 *$-weighted gradient echo (T2*GRE) and susceptibility-weighted imaging (SWI) and diffusion-weighted imaging (DWI) that is sensitive for intra- and extracellular edema can confirm the DAI diagnosis by demonstrating lesions in the white matter of cerebral hemispheres, corpus callosum, and brainstem. ${ }^{11-13}$

The prognostic methods to assess long-term outcome after DAI are still unreliable. The presence and number of hemorrhagic and nonhemorrhagic lesions detected by DAI-sensitive sequences on MRI have shown correlation with outcome,${ }^{14-17}$ but the prognostic value of anatomical lesion location has not been analyzed extensively. Hemorrhagic and nonhemorrhagic DAI-associated lesions on MRI seem to diminish over time, ${ }^{17,18}$ and significant prognostic information may therefore be lost when late MRI is used. ${ }^{17}$ Because early MRI can be challenging to perform in unstable severely traumatized patients with TBI, there is a paucity of studies that evaluate the value of MRI in the early phase of severe TBI with DAI for prediction of long-term outcome. Therefore the aims of this study were to:

1. Quantify and assess the anatomical distribution of lesions associated with DAI comparing different MRI sequences.

2. Apply the Adams anatomical grading system based on histopathology ${ }^{7}$ using modern MRI sequences.

3. Evaluate whether anatomical localization of lesions and MRI grading provide valuable prognostic information in combination with clinical prognostic factors.

4. Propose a new MRI grading system based on the Adams anatomical histopathological grading system and clinical prognostic factors to improve prediction of long-term outcome.

\section{Methods}

\section{Patient selection}

The criteria for inclusion were: age more than 15 years, severe head injury with post-resuscitation Glasgow Coma Scale (GCS) score $<9$ or Glasgow Motor Score (GMS) $<6$, no mass lesion over $25 \mathrm{cc}$, no ischemic/vascular lesion explaining the depressed level of consciousness, and DAI-associated lesions confirmed on MRI performed within 1 week after trauma. During the study period between January 2006 and February 2014, a total of 59 patients older than 15 years were admitted to the neurointensive care (NIC) unit with suspected DAI on the initial CT.

In 20 patients, MRI was not performed to confirm the DAI diagnosis, and 9 patients were investigated with MRI in the subacute phase beyond 1 week post-injury. The main reasons for not performing MRI acutely were externally fixated extremity fractures incompatible with MRI followed by intracranial pressure (ICP) instability, which made transfer from the NIC unit unsafe. The remaining 30 patients with severe TBI included in the study had clinical and CT indications of DAI, and this diagnosis was confirmed with MRI within 1 week of the injury.

With the exception of one patient with grade II thrombocytopenia (platelet count of $50-75 \times 10^{9} / \mathrm{L}$ ), no patient had a coagulopathy or was receiving anticoagulant therapy before the trauma.
Low-dose antithrombotic therapy with low-molecular weight heparin (LMWH; Dalteparin 2500-5000 units) was administered to $16 / 30$ patients at mean 6 days (standard deviation $[\mathrm{SD}] \pm 4$ days) post-trauma. The LMWH was administered after acquisition of MRI images in all but two patients, in whom LMWH was administered 1 day before the MRI.

\section{Neurointensive care}

All patients in the presented study were treated at the NIC unit at the Uppsala University Hospital. The patients were ventilated endotracheally and sedated using a combination of intermittent intravenous (IV) morphine analgesia (Morfin Meda 1-3 mg, Meda, Sollentuna, Sweden) and continuous IV propofol infusion (Propofol-Lipuro 1-4 mg/kg/h, B. Braun Melsungen AG, Melsungen, Germany). All patients had ICP monitoring (Codman ICP express intraparenchymal monitor in 22 patients, external ventricular drain in 8 patients) for continuous measurements of ICP and cerebral perfusion pressure (CPP).

Patients with TBI were treated using an ICP- and CPP-guided standard protocol including mild hyperventilation initially $\left(\mathrm{PaCO}_{2}\right.$ 30-35 mm Hg; 4.0-4.5 kPa), 30-degree elevated head of bed, and volume expansion to normovolemia and a central venous pressure of $0-5 \mathrm{~mm} \mathrm{Hg} .{ }^{19}$ To minimize secondary brain injury, the aim of treatment was to keep ICP at $<20 \mathrm{~mm} \mathrm{Hg}$ and CPP at $>60 \mathrm{~mm} \mathrm{Hg}$ according to an organized secondary insult program. ${ }^{19}$ ICP elevations not controlled by standard therapy or CSF drainage were treated with pharmacologically induced coma using first continuous propofol infusions without neurological wake-up tests ${ }^{20}$ and as second-tier therapy, continuous sodium pentobarbital infusion therapy and/or a decompressive craniotomy.

\section{Imaging and image analysis}

An initial CT was performed on admission and scored according to the Marshall classification by the first author (SAH). ${ }^{8}$ MRI was performed as soon as the patients were medically stable. MRI was performed with a 1.5T scanner (Siemens Avanto, Siemens Medical, Erlangen, Germany). Imaging included a gradient echo T2*GRE sequence (repetition time [TR]: $500 \mathrm{msec}$, echo time [TE]: $14 \mathrm{msec}$, flip angle [FA]: 30 degrees, acquisition voxel: $0.9 \times 0.9 \times 3 \mathrm{~mm}$ ); a diffusion-weighted (DW) sequence (SE-EPI, b-value $1000 \mathrm{~s} / \mathrm{mm}^{2}$, TR: $4700 \mathrm{msec}$, TE: $89 \mathrm{msec}$, acquisition voxel: $1.2 \times 1.2 \times 5 \mathrm{~mm}$ ); and a SWI sequence (TR: $49 \mathrm{msec}$, TE: $40 \mathrm{msec}$, FA: 15 degrees, acquisition voxel: $0.9 \times 0.9 \times 1.5 \mathrm{~mm}$ ).

The images were assessed independently by two of the authors (SAH and JW). DAI-associated lesions were defined as hypointense/decreased signals for T2*GRE and SWI sequences, and/ or restricted diffusion for DW sequence in white matter structures not extending to the cortex. No size limit was used. Present small cortical lesions were excluded. Mimicking lesions such as vascular structures were excluded based on tubular shape.

The Adams anatomical histopathological grading system was applied. ${ }^{7}$ The grading was performed both for each sequence separately and for all MRI sequences combined, where patients were classified according to the sequence giving the highest grade. The number of DAI-associated lesions was counted on each side of the midline in different anatomical localizations of the brain as presented in Table 1.

The mesencephalon was anatomically divided into three regions; (1) crus cerebri, (2) substantia nigra and mesencephalic tegmentum, and (3) tectum including the superior and inferior colliculi (Fig. 1). The pons and the medulla oblongata were anatomically divided into a ventral and a dorsal tegmental portion. For practical reasons, patients with $>10$ lesions in one anatomical location unilaterally were assigned the number 15 , similar to the approach of an earlier study. ${ }^{17}$ Both raters were blinded to patients' outcome. 
Table 1. Protocol Used for Counting Lesions in Different Anatomical Localizations Using Magnetic Resonance Imaging in Patients with Diffuse Axonal Injury

\begin{tabular}{llllllll}
$\begin{array}{l}\text { Grade } 1 \\
\text { (hemispheric) }\end{array}$ T2*GRE Right & Left & DW & Right & Left & SWI & Right \\
\hline
\end{tabular}

Frontal

Temporal

Parietal

Occipital

Insular

Cerebellar

Grade 2 (corpus

callosum) $\quad T 2 * G R E \quad$ DW $\quad S W I$

Genu

Body

Splenium

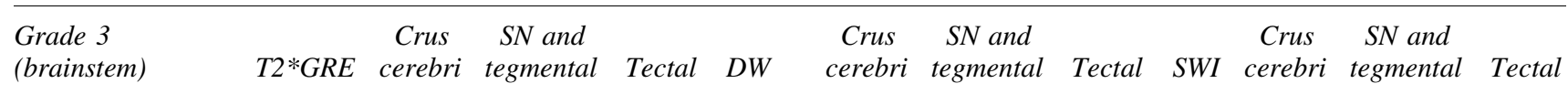

Mesencephalon

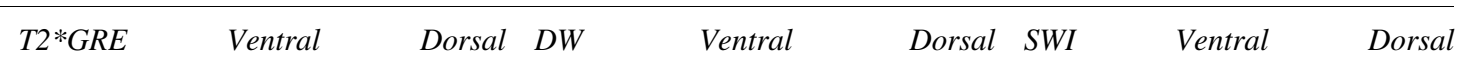

Pons

Medulla oblongata

\begin{tabular}{|c|c|c|c|c|c|c|c|c|c|}
\hline $\begin{array}{l}\text { Deep hemispheric } \\
\text { lesions }\end{array}$ & $T 2^{*} G R E$ & Right & Left & $D W$ & Right & Left & $S W I$ & Right & Left \\
\hline
\end{tabular}

Thalamus

Basal ganglia

Internal capsule

T2*GRE, T2*-weighted gradient echo; DW, diffusion-weighted sequence; SWI, susceptibility-weighted imaging;

\section{Potential clinical prognostic predictors}

Demographic data and other clinical information were collected from the electronic patient record system, the TBI NIC database, and the Uppsala TBI registry (www.ucr.uu.se). Physiologic data were collected using a computerized multimodality monitoring system collecting minute-by-minute average values for each monitored parameter. ${ }^{21}$ Data were manually assessed, and invalid data were withdrawn from the total monitoring time. Physiological data collection was interrupted when the patient was taken to the operating room or for radiologic evaluation as well as when network or software failures occurred.

The remaining monitoring data, after removal of invalid data and without the gaps from interruption of monitoring, gave the good
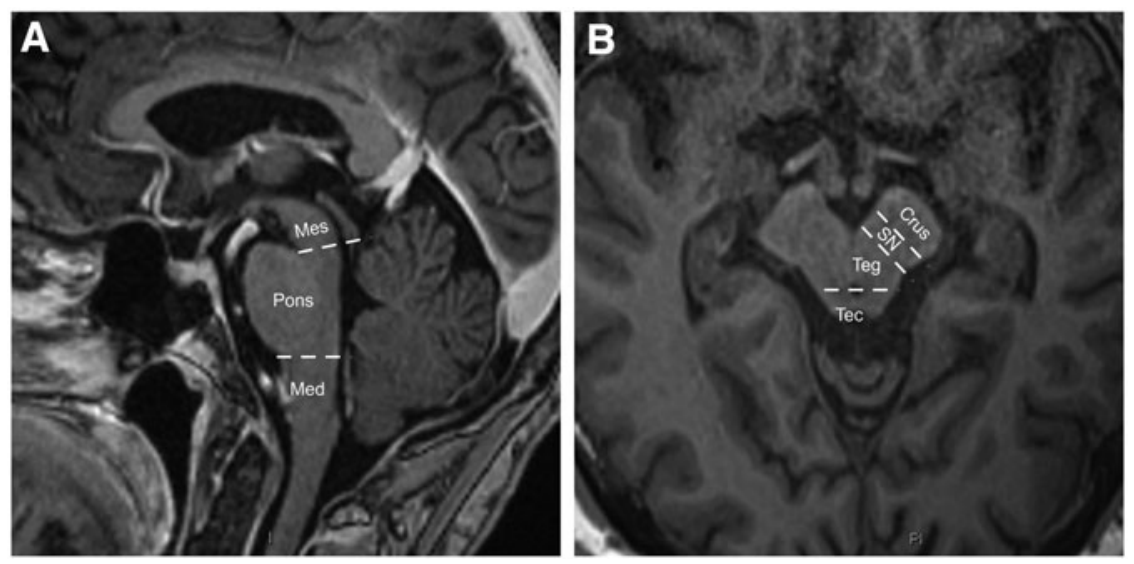

FIG. 1. (A, B) Definition of brainstem and mesencephalic structures. The mesencephalon was anatomically divided into three regions; region (1) crus cerebri, (2) substantia nigra and tegmentum, and (3) tectum including the superior and inferior colliculi, Mes, mesencephalon; Med, medulla oblongata; Crus, crus cerebri; SN, substantia nigra; Teg, mesencephalic tegmentum; Tec, mesencephalic tectum. 
Table 2. Demographic Data and Potential Prognostic Clinical Factors and Their Association with Long-Term Outcome (Glasgow Outcome Scale Extended) in Ordinal Univariate Logistic Regression

\begin{tabular}{|c|c|c|c|}
\hline Variable & No of cases (\%) & OR, $95 \% C I$ & $\mathrm{p}$ value ${ }^{\S}$ \\
\hline Age (years)* & $31.2(16-60)$ & $1.10,1.04-1.17$ & 0.001 \\
\hline Sex (male/female) & $26 / 4(86.7 / 13.3)$ & $1.05,0.41-2.68$ & 0.91 \\
\hline Length of stay* (days) & $11.8(3-30)$ & $1.07,0.96-1.20$ & 0.21 \\
\hline \multicolumn{4}{|l|}{ Injury mechanism } \\
\hline MVA & $18(60)$ & $0.46,0.12-1.68$ & 0.24 \\
\hline Sport & $7(23.3)$ & $0.29,0.07-1.32$ & 0.11 \\
\hline Fall & $4(13.3)$ & $1.25,0.23-6.62$ & 0.80 \\
\hline Assault & $1(3.3)$ & $R$ & $R$ \\
\hline Admission GMS score ${ }^{\dagger}$ & $5(6-1)$ & 14.6, 2.58-83.22 & 0.0025 \\
\hline Motor deficit $(\mathrm{y} / \mathrm{n})$ & & $1.34,0.70-2.57$ & 0.38 \\
\hline None & $14(46.7)$ & & \\
\hline Extremity & $7(23.3)$ & & \\
\hline Hemiparesis & $8(26.7)$ & & \\
\hline Tetraparesis & $1(3.3)$ & & \\
\hline Extracranial injury & $22(73.3)$ & $2.04,0.95-4.39$ & 0.07 \\
\hline ISS $*$ & $24.3(16-45)$ & $1.03,0.95-1.11$ & 0.43 \\
\hline \multicolumn{4}{|l|}{ Marshall score } \\
\hline DI I & $3(10)$ & $R$ & $R$ \\
\hline DI II & $22(73.3)$ & $1.63,0.59-4.52$ & 0.34 \\
\hline DI III & $5(16.7)$ & $1.85,0.51-6.71$ & 0.34 \\
\hline \multicolumn{4}{|l|}{ ICP management } \\
\hline Basal treatment & $24(80)$ & $R$ & $R$ \\
\hline CSF drainage & $3(10)$ & $1.31,0.28-6.18$ & 0.73 \\
\hline Propofol coma & $3(10)$ & $2.33,0.47-11.49$ & 0.30 \\
\hline GMS score at discharge $^{\dagger}$ & $6(6-4)$ & $10.32,2.75-38.75$ & 0.0005 \\
\hline \multicolumn{4}{|l|}{ GOSE $>6$ months } \\
\hline $1-2$ & $1(3.3)$ & & \\
\hline $3-4$ & $8(26.7)$ & & \\
\hline $5-6$ & $11(36.7)$ & & \\
\hline $7-8$ & $9(30)$ & & \\
\hline $\mathrm{LF}$ & $1(3.3)$ & & \\
\hline
\end{tabular}

${ }^{\S}$ Association with Glasgow Outcome Scale extended.

* Listed as mean and range.

${ }^{\dagger}$ Listed as median and range.

OR, odds ratio; CI, confidence interval; MVA, motor vehicle accident; $R$, reference category; GMS, Glasgow Motor Score; ISS, Injury Severity

Score $^{49}$; DI, diffuse injury; ICP, intracranial pressure; CSF, cerebrospinal fluid; LF, lost to follow-up.

GOSE, Glasgow Outcome Scale extended.

monitoring time (GMT), which was assessed for ICP, CPP, mean arterial blood pressure (MAP), heart rate, and core body temperature. GMT for the first $96 \mathrm{~h}$ after start of monitoring was used for analysis, and a minimum of $24 \mathrm{~h}$ of GMT was required for inclusion.

Thresholds for secondary insults were defined based on the treatment goals for ICP, CPP, MAP, heart rate, and core body temperature and included ICP $>20 \mathrm{~mm} \mathrm{Hg}, \mathrm{CPP}<60 \mathrm{~mm} \mathrm{Hg}$, MAP $<70 \mathrm{~mm} \mathrm{Hg}$, heart rate $>120 \mathrm{bpm}$, and core body temperature $>38^{\circ} \mathrm{C}$. The amounts of secondary insults were calculated as the proportion of GMT at the insult level and are presented as percent of GMT. Mean pressure reactivity index (PRx), the moving correlation coefficient between fluctuations in ICP and arterial blood pressure $(\mathrm{ABP})$ was calculated as a measure of the status of cerebral pressure autoregulation during the same period. ${ }^{22} \mathrm{We}$ also calculated mean ICP amplitude to evaluate intracranial compliance. ${ }^{23}$

\section{Outcome}

Long-term outcome was assessed at $>6$ months after injury according to the extended Glasgow Outcome Scale (GOSE). The GOSE score was assessed by an intensive care specialist nurse who was trained to perform the structured interview and blinded to all clinical data. Evaluation was performed via a standardized ques- tionnaire based on the structured interviews proposed by Wilson and colleagues, ${ }^{24}$ to be filled out by patients or the closest relative. Patients or relatives were contacted by telephone when supplementary information was needed.

\section{Statistical methods}

The inter-rater agreement was calculated for agreement of DAI grading between the two independent evaluators for all sequences and for substantia nigra or tegmental/tectal brainstem lesion on SWI. Inter-rater agreement for total number of DAI-associated lesions was also calculated for all three sequences. Disagreement was settled with a mutual evaluation of images by SAH and JW, and the agreed consensus was used for data analysis.

The inter-rater agreement was analyzed using linear weighted Cohen $\kappa$ statistics for categorical values, and the intra-class correlation coefficient (ICC) was calculated with two-way random single measures (consistency/absolute agreement) for continuous values. Values of $<0.2$ were considered as poor, $0.21-0.4$ as fair, $0.41-0.6$ as moderate, $0.61-0.8$ as good, and $>0.81$ as very good agreement for both $\kappa$ and ICC values.

Statistica 12.0 (Stat Soft, Inc. Tulsa, OK) was used for descriptive and analytical statistics. The Student $t$ test was used for normal 
distributed data and the Fisher exact test for comparison of proportions. The Mann-Whitney $U$ test was used for skewed distributions. The association between potential prognostic factors and long-term outcome was analyzed with ordinal logistic regression with GOSE category as the dependent variable. Factors found significantly associated with outcome on univariate analysis were further analyzed in multivariate analysis with ordinal logistic regression. A $p$ value of $<0.05$ was considered statistically significant.

\section{Ethics}

The Uppsala University Regional Ethical Review Board for clinical research granted permission for the study.

\section{Results}

Demographics, injury related variables, ICP management, and outcomes are presented in Table 2 . Age was significantly associated with long-term outcome $(p=0.001$, odds ratio [OR] $1.10,95 \%$ confidence interval [CI] 1.04-1.17) as were GMS at admission ( $p=0.0025$, OR 14.6, 95\% CI 2.58-83.22) and GMS at discharge $(p<0.001$, OR $10.32,95 \%$ CI 2.75-38.75) on univariate analysis.

\section{Anatomical distribution and univariate analysis for outcome prediction}

Anatomical distributions of DAI-associated lesions are shown in Table 3. SWI images identified significantly more hemorrhagic lesions than $\mathrm{T} 2 * \mathrm{GRE}$ images in cerebral hemispheres $(p<0.001)$, corpus callosum $(p=0.0026)$, brainstem $(p=0.024)$, and in total $(p<0.001)$. A higher number of lesions were also found when the SWI sequence was compared with the DW sequence in cerebral hemispheres $(p<0.001)$, corpus callosum $(p=0.001)$, brainstem $(p=0.009)$, thalamus, basal ganglia and internal capsule $(p<0.001)$, and in total $(p<0.001)$. No significant difference was found when lesion numbers identified with T2*GRE and DW sequence were compared.

With the T $2 *$ GRE sequence, neither anatomical distribution nor the total lesion load was significantly associated with poor longterm outcome. Significant associations with long-term outcome in univariate analysis were seen for hemorrhagic lesions identified on SWI in the region corresponding to the substantia nigra and tegmentum of the mesencephalon, in the region corresponding to the tectum of the mesencephalon ( $p=0.005$, OR 2.46, 95\% CI $1.31-$ 4.61 and $p=0.01$, OR 2.81, 95\% CI 1.26-6.27), and in the dorsal pons $(p=0.04$, OR $1.76,95 \%$ CI $1.03-2.99)$. With the DW sequence, only lesions in the thalamus, basal ganglia, and internal capsule were significantly associated with long-term outcome ( $p=0.025$, OR $2.59,95 \%$ CI 1.13-5.94).

Bilateral brainstem lesions were identified in $2 / 9$ patients with brainstem lesions with the T2*GRE sequence, in $3 / 12$ patients with brainstem lesions with the DW sequence, and in 10/16 patients with brainstem lesions with the SWI sequence. A significant association with long-term outcome was seen for hemorrhagic bilateral brainstem lesions with SWI ( $p=0.03$, OR 2.42, 95\% CI 1.09-5.38).

\section{Anatomical DAl grading}

Distribution in Adams anatomical DAI grade using MRI is presented in Table 4. Twenty-one of $30(70 \%)$ patients were grade 3 when patients were classified according to the sequence giving the highest grade. For hemorrhagic lesions, the SWI sequence identified significantly more DAI grade III patients than T2*GRE $(p=0.006)$. The overall DAI grade according to Adams was not significantly associated with long-term outcome neither using the $\mathrm{T} 2 * \mathrm{GRE}, \mathrm{DW}$, or SWI sequences alone nor when the sequences were combined.

\section{Inter-rater agreement}

For DAI grading and for classification of substantia nigra or tegmental/tectal lesions in the mesencephalon, overall agreement was very good with Cohen $\kappa>0.8$, (mean 0.90, range 0.82-0.91). Intraclass correlation (ICC) calculations showed good agreement for quantification of total DAI-associated lesion load on all three sequences with ICC values $>0.6$ (mean 0.66 , range $0.62-0.68$ ).

\section{NIC monitoring data}

Monitoring data were available in 27 patients for MAP and heart rate, in 25 patients for ICP and CPP, and in 24 patients for body temperature. Mean PRx and ICP amplitude values were available in 24 patients. Mean proportion of GMT with ICP $>20 \mathrm{~mm} \mathrm{Hg}$ was 2.8 $\%(\mathrm{SD} \pm 3.3 \%)$ during the first 5 days. The proportion of GMT with ICP $>20 \mathrm{~mm} \mathrm{Hg}$ was not associated with long-term outcome. Mean proportion of GMT with CPP $<60 \mathrm{~mm} \mathrm{Hg}$ was $3.8 \%$ ( $\mathrm{SD} \pm 3.5 \%$ ). Proportion of GMT at CPP $<60 \mathrm{~mm} \mathrm{Hg}$ had a significant relation with long-term outcome, with better outcomes in patients with higher proportions of GMT at CPP $<60 \mathrm{~mm} \mathrm{Hg}(p=0.007$, OR $0.70,95 \%$ CI $0.54-0.91$ ) in univariate analysis.

Mean proportion of GMT was $2.8 \%$ (SD $\pm 2.8 \%$ ) for MAP, $<70 \mathrm{~mm} \mathrm{Hg}, 0.4 \%(\mathrm{SD} \pm 0.5 \%)$ for heart rate $>120 \mathrm{bpm}$, and $15.8 \%$ (SD $\pm 14.9 \%$ ) for body temperature $>38^{\circ} \mathrm{C}$. The proportion of GMT with MAP $<70 \mathrm{~mm} \mathrm{Hg}$, heart rate $>120 \mathrm{bpm}$, and body temperature $>38^{\circ} \mathrm{C}$ was not associated with long-term outcome.

Mean ICP amplitude in all patients was $5.6 \mathrm{~mm} \mathrm{Hg}(\mathrm{SD} \pm 2.0 \mathrm{~mm}$ $\mathrm{Hg}$ ), indicating generally low brain compliance, but ICP amplitude had no association with long-term outcome. Mean PRx during the first 5 days was 0.007 ( $S D \pm 0.09$ ). Six patients had a PRx more than 0.1 , indicating possibly disturbed cerebrovascular pressure reactivity. Overall, there was little variation in PRx for these patients, and no association with long-term outcome was found.

\section{Multivariate analysis for prognostic predictors of outcome}

Factors significantly associated with long-term outcome on univariate analysis (Table 3) were used for multivariate analysis in ordinal logistic regression with the GOSE after 6 months as the dependent variable. The model yielded two factors with significant independent association with poor long-term outcome: number of lesions in the thalamus, basal ganglia, and internal capsule on the DW sequence ( $p=0.039$, OR 2.76, 95\% CI 1.05-7.22) and number of lesions in the substantia nigra and mesencephalic tegmentum on SWI $(p=0.01$, OR $2.31,95 \%$ CI 1.22-4.37). A new model (Table 5) with these two MRI factors was computed including prognostic clinical factors found to be significantly associated with long-term outcome in the univariate analysis; i.e., age, GMS at admission, GMS at discharge, and proportion of GMT with CPP $<60 \mathrm{~mm} \mathrm{Hg}$.

CT Marshall Score and overall DAI grade on MRI were also inserted in the logistic regression model. Higher age $(p=0.005$, OR $1.18,95 \%$ CI 1.05-1.32), and number of lesions in the substantia nigra and mesencephalic tegmentum on SWI ( $p=0.008$, OR 4.51, $95 \%$ CI 1.48-13.78) were the only factors found to have an independent association with worse long-term outcome in the combined multiple logistic regression model (Table 5). 
Table 3. Anatomical Distribution and Number of Diffuse Axonal Injury Associated Lesions and Their Association with Long-Term Outcome (Glasgow Outcome Scale Extended) in Ordinal Univariate Logistic Regression

\begin{tabular}{|c|c|c|c|c|c|c|}
\hline & Mean & Minimum & Maximum & $S D$ & $O R, 95 \% C I$ & $\mathrm{p}$ value ${ }^{\S}$ \\
\hline \multicolumn{7}{|l|}{$T 2 * G R E$} \\
\hline \multicolumn{7}{|l|}{ Grade 1} \\
\hline Total cerebral hemispheric ${ }^{\Delta}$ & 12.3 & 0 & 44 & 10.0 & $1.26,0.96-1.09$ & 0.46 \\
\hline Total cerebellar & 0.3 & 0 & 3 & 0.8 & $0.51,0.19-1.35$ & 0.18 \\
\hline \multicolumn{7}{|l|}{ Grade 2} \\
\hline \multicolumn{7}{|l|}{ Grade 3} \\
\hline Total brainstem $^{\varphi}$ & 0.9 & 0 & 7 & 1.7 & & \\
\hline Total mesencephalon & 0.5 & 0 & 6 & 1.2 & & \\
\hline Crus cerebri & 0.1 & 0 & 1 & 0.3 & $0.72,0.09-6.06$ & 0.76 \\
\hline SN and mesencephalic tegmentum & 0.3 & 0 & 5 & 1.0 & $0.97,0.50-1.86$ & 0.92 \\
\hline Mesencephalic tectum & 0.2 & 0 & 2 & 0.5 & $1.94,0.15,25.48$ & 0.61 \\
\hline Total pons & 0.3 & 0 & 4 & 0.8 & & \\
\hline Pons ventral & 0.1 & 0 & 2 & 0.4 & $0.64,0.12-3.26$ & 0.59 \\
\hline Pons dorsal (tegmentum) & 0.3 & 0 & 2 & 0.6 & $0.65,0.16,2.70$ & 0.55 \\
\hline Total medulla oblongata & 0.1 & 0 & 3 & 0.6 & & \\
\hline Medulla oblongata ventral & 0 & 0 & 0 & 0 & N/A & N/A \\
\hline Medulla oblongata dorsal (tegmentum) & 0.1 & 0 & 3 & 0.6 & $0.68,0.20-2.31$ & 0.54 \\
\hline \multicolumn{7}{|l|}{ Deep hemispheric lesions } \\
\hline Thalamus, BG, IC & 2.9 & 0 & 11 & 2.8 & $1.11,0.89-1.37$ & 0.36 \\
\hline Total lesion load & 19.2 & 3 & 49 & 13.8 & $1.02,0.96-1.08$ & 0.49 \\
\hline \multicolumn{7}{|l|}{$D W$} \\
\hline Total cerebral hemispheric ${ }^{\Delta}$ & 9.0 & 0 & 40 & 8.5 & $1.04,0.96-1.13$ & 0.32 \\
\hline Total cerebellar & 0.3 & 0 & 4 & 0.8 & $0.92,0.40-2.09$ & 0.84 \\
\hline \multicolumn{7}{|l|}{ Grade 2} \\
\hline Total corpus callosum ${ }^{\Omega}$ & 2.6 & 0 & 12 & 2.5 & $1.01,0.78-1.31$ & 0.92 \\
\hline \multicolumn{7}{|l|}{ Grade 3} \\
\hline Total brainstem ${ }^{\varphi}$ & 0.8 & 0 & 3 & 1.0 & & \\
\hline Total mesencephalon & 0.4 & 0 & 2 & 0.7 & & \\
\hline Crus cerebri & 0 & 0 & 0 & 0 & N/A & N/A \\
\hline $\mathrm{SN}$ and mesencephalic tegmentum & 0.2 & 0 & 1 & 0.4 & $0.67,0.10-4.38$ & 0.68 \\
\hline Mesencephalic tectum & 0.2 & 0 & 2 & 0.5 & $0.73,0.19-2.81$ & 0.65 \\
\hline Total pons & 0.4 & 0 & 2 & 0.6 & & \\
\hline Pons ventral & 0.07 & 0 & 1 & 0.3 & $15.0,0.7-312$ & 0.08 \\
\hline Pons dorsal (tegmentum) & 0.3 & 0 & 2 & 0.6 & $2.32,0.67-7.96$ & 0.19 \\
\hline Total medulla oblongata & 0 & 0 & 0 & 0 & & \\
\hline Medulla oblongata ventral & 0 & 0 & 0 & 0 & N/A & N/A \\
\hline Medulla oblongata dorsal (tegmentum) & 0 & 0 & 0 & 0 & N/A & N/A \\
\hline \multicolumn{7}{|l|}{ Deep hemispheric lesions } \\
\hline Thalamus, BG, IC & 1.2 & 0 & 5 & 1.4 & 2.59, 1.13-5.94 & 0.025 \\
\hline Total lesion load & 13.8 & 1 & 45 & 10.0 & $1.03,0.97-1.10$ & 0.33 \\
\hline \multicolumn{7}{|l|}{ SWI } \\
\hline \multicolumn{7}{|l|}{ Grade 1} \\
\hline Total cerebral hemispheric ${ }^{\Delta}$ & 28.8 & 0 & 68 & 17.2 & $1.01,0.96-1.06$ & 0.65 \\
\hline Total cerebellar & 1.0 & 0 & 7 & 2.0 & $1.26,0.87-1.82$ & 0.22 \\
\hline \multicolumn{7}{|l|}{ Grade 2} \\
\hline Total corpus callosum ${ }^{\Omega}$ & 7.1 & 0 & 21 & 6.4 & $0.99,0.89-1.11$ & 0.92 \\
\hline \multicolumn{7}{|l|}{ Grade 3} \\
\hline Total brainstem $^{\varphi}$ & 3.9 & 0 & 17 & 4.2 & & \\
\hline Total mesencephalon & 2.7 & 0 & 12 & 3.3 & & \\
\hline Crus cerebri & 1.1 & 0 & 6 & 1.7 & $1.70,0.99-2.91$ & 0.05 \\
\hline $\mathrm{SN}$ and mesencephalic tegmentum & 1.0 & 0 & 5 & 1.6 & $2.46,1.31-4.61$ & 0.005 \\
\hline Mesencephalic tectum & 0.6 & 0 & 5 & 1.2 & $2.81,1.26-6.27$ & 0.01 \\
\hline Total pons & 1.1 & 0 & 5 & 1.6 & & \\
\hline Pons ventral & 0.1 & 0 & 2 & 0.4 & $0.73,0.14-3.76$ & 0.71 \\
\hline Pons dorsal (tegmentum) & 1.0 & $\mathbf{0}$ & 5 & 1.5 & $1.76,1.03-2.99$ & 0.04 \\
\hline
\end{tabular}


TABle 3. (CONTINUEd)

\begin{tabular}{|c|c|c|c|c|c|c|}
\hline & Mean & Minimum & Maximum & $S D$ & $O R, 95 \% C I$ & $\mathrm{p}$ value ${ }^{\S}$ \\
\hline Total medulla oblongata & 0.2 & 0 & 3 & 0.7 & & \\
\hline Medulla oblongata ventral & 0 & 0 & 0 & 0 & N/A & N/A \\
\hline Medulla oblongata dorsal (tegmentum) & 0.2 & 0 & 3 & 0.7 & $0.93,0.29-2.91$ & 0.90 \\
\hline \multicolumn{7}{|l|}{ Deep hemispheric lesions } \\
\hline Thalamus, BG, IC & 9.4 & 0 & 32 & 7.2 & $1.11,0.90-1.37$ & 0.33 \\
\hline Total lesion load $\varnothing$ & 44.9 & 11 & 85 & 21.1 & $1.02,0.99-$ & 0.23 \\
\hline
\end{tabular}

${ }^{\Delta}$ Sum of lesions on both sides of midline in lobes of cerebral hemispheres.

${ }^{9}$ Sum of lesions on both sides of midline in cerebellum.

${ }^{\Omega}$ Sum of lesions in genu, body and splenium of corpus callosum.

${ }^{\varphi}$ Sum of lesions in all regions of the brainstem.

${ }^{\emptyset_{\text {Sum }}}$ of all regions of brain on both sides of midline.

${ }^{\S}$ Association with Glasgow Outcome Scale extended.

$\mathrm{SD}$, standard deviation; OR, odds ratio; CI, confidence interval; T2*GRE, T2*-weighted gradient echo; SN, substantia nigra; DW, diffusion-weighted sequence; SWI, susceptibility-weighted imaging; BG, basal ganglia; IC, internal capsule; N/A, not available.

For the development of a new MRI classification system, we also performed a multivariate analysis with factors found significantly associated with long-term outcome but with dichotomized age $\geq /<30$ years and presence or no presence of hemorrhagic lesions in the substantia nigra and mesencephalic tegmentum (Table 6). This was made to enable a classification system that is simple to use.

The age division was chosen based on the age distribution of the patient material ( $n=17<30$ years and $n=13 \geq 30$ years). Similar to the previous ordinal logistic regression above, we found that age $\geq 30$ years ( $p=0.011$, OR 3.64, 95\% CI 1.35-9.84) and the presence of hemorrhagic lesions in the substantia nigra and mesencephalic tegmentum ( $p=0.012$, OR $3.69,95 \%$ CI $1.33-10.2)$ were the only factors with independent predictive value for a worse long-term outcome (Table 6).

Table 4. Distribution of the Adams Anatomical Grades Using Different Magnetic Resonance Imaging Sequences in 30 Patients with Severe Diffuse Axonal Injury*

\begin{tabular}{cccc}
\hline MRI sequence & No of cases (\%) & OR, 95\% CI & p value \\
\hline T2* & Available in 27 cases & & \\
Grade I & $7(25.9)$ & $R$ & $R$ \\
Grade II & $11(40.7)$ & $0.67,0.27-1.69$ & 0.40 \\
Grade III & $9(33.3)$ & $1.07,0.27-1.69$ & 0.89 \\
DW & Available in 29 cases & & \\
Grade I & $5(17.2)$ & $R$ & $R$ \\
Grade II & $12(41.4)$ & $0.80,0.31-2.03$ & 0.64 \\
Grade III & $12(41.4)$ & $0.97,0.39-2.43$ & 0.95 \\
SWI & Available in 25 cases & & \\
Grade I & $1(4)$ & $R$ & $R$ \\
Grade II & $8(32)$ & $0.45,0.09-2.11$ & 0.31 \\
Grade III & $16(64)$ & $3.30,0.77-14.19$ & 0.11 \\
Total grade & & & \\
Grade I & $2(6.7)$ & $R$ & $R$ \\
Grade II & $7(23.3)$ & $0.68,0.19-2.45$ & 0.55 \\
Grade III & $21(70)$ & $1.52,0.52-4.48$ & 0.45 \\
\hline
\end{tabular}

*Association with long-term outcome (Glasgow coma scale extended [GOSE]) in ordinal univariate logistic regression.

${ }^{\dagger}$ Diffuse axonal injury grade when lesions in all sequences are combined ${ }^{\S}$ Association with GOSE.

$\mathrm{OR}$, odds ratio; CI, confidence interval; $R$, reference category; $2 * \mathrm{GRE}$ T2*-weighted gradient echo; DW, diffusion-weighted sequence; SWI, susceptibility-weighted imaging;

\section{Discussion}

DAI was first described by Strich and colleagues ${ }^{25}$ in 1956 and later characterized by the work of Adams and coworkers ${ }^{7}$ and Gennarelli and associates, ${ }^{9}$ linking the histopathological features of post-traumatic widespread damage of axons to angular or rotational acceleration-deceleration forces to the head causing stretching injury to brain tissue. MRI is the imaging modality of choice to detect hemorrhagic and nonhemorrhagic DAI-associated lesions in the clinical setting. In this study, a cohort of patients with severe TBI with DAI was examined with early MRI within 1 week after trauma, and correlation with outcome measured by GOSE after 6 months was assessed.

\section{MRI sequences}

The study included three MRI sequences evaluated in patients with severe TBI and DAI. DWI visualizes nonhemorrhagic lesions while T2*GRE and SWI both visualize hemorrhagic lesions. The SWI sequence is more effective in demonstrating susceptibility differences between tissues and therefore more sensitive to cerebral

Table 5. Odds Ratio Multiple Ordinal logistic Regression, Dependent Glasgow Outcome SCAle Extended

\begin{tabular}{lcccc}
\hline & & & & \\
& $\begin{array}{c}\text { Regression } \\
\text { coefficient }\end{array}$ & $\begin{array}{c}\text { Odds } \\
\text { ratio }\end{array}$ & $\begin{array}{c}\text { Confidence } \\
\text { interval }\end{array}$ & p value \\
\hline Age & $\mathbf{0 . 1 6}$ & $\mathbf{1 . 1 8}$ & $\mathbf{1 . 0 5}-\mathbf{1 . 3 2}$ & $\mathbf{0 . 0 0 5}$ \\
$\begin{array}{l}\text { Lesions in SN and } \\
\text { mesencephalic }\end{array}$ & $\mathbf{1 . 5 1}$ & $\mathbf{4 . 5 1}$ & $\mathbf{1 . 4 8}-\mathbf{1 3 . 7 8}$ & $\mathbf{0 . 0 0 8}$ \\
$\quad$ tegmentum on SWI & & & & \\
$\begin{array}{l}\text { Thalamic, BG, IC } \\
\text { lesions on DW }\end{array}$ & & & & N.S \\
$\begin{array}{l}\text { Adams DAI grade } \\
\text { Marshall score }\end{array}$ & & & & \\
GMS at admission & & & & N.S \\
GMS at discharge & & & & N.S \\
CPP <60 mm Hg* & & & N.S \\
\hline
\end{tabular}

*Proportion of good monitoring time during first 5 days.

SN, substantia nigra; SWI, susceptibility-weighted imaging; BG, basal ganglia; IC, internal capsule; DW, diffusion-weighted sequence; DAI, diffuse axonal injury; GMS, Glasgow Motor Score; CPP, cerebral perfusion pressure. 
Table 6. Odds Ratio Multiple Ordinal Logistic Regression, Dependent Glasgow Outcome SCALE EXTENDED

\begin{tabular}{lcccc}
\hline & $\begin{array}{c}\text { Regression } \\
\text { coefficient }\end{array}$ & $\begin{array}{c}\text { Odds } \\
\text { ratio }\end{array}$ & $\begin{array}{c}95 \% \text { confidence } \\
\text { interval }\end{array}$ & $\begin{array}{c}\mathrm{p} \\
\text { value }\end{array}$ \\
\hline Age $\geq 30$ & $\mathbf{1 . 2 9}$ & $\mathbf{3 . 6 4}$ & $\mathbf{1 . 3 5} \mathbf{- 9 . 8 4}$ & $\mathbf{0 . 0 1 1}$ \\
$\begin{array}{l}\text { Lesions in SN and } \\
\text { mesencephalic }\end{array}$ & $\mathbf{1 . 3 0}$ & $\mathbf{3 . 6 9}$ & $\mathbf{1 . 3 3}-\mathbf{1 0 . 2}$ & $\mathbf{0 . 0 1 2}$ \\
$\quad$ tegmentum yes/no & & & & \\
Adams DAI grade & & & & N.S \\
Marshall score & & & & N.S \\
GMS at admission & & & & N.S \\
GMS at discharge & & & & N.S \\
CPP $>60$ mm Hg** & & & & N.S \\
\hline
\end{tabular}

*Age $\geq<30$ years and lesions in the substantia nigra and mesencephalic tegmentum yes/no dichotomized.

***Proportion of good monitoring time during first 5 days.

SN, substantia nigra; DAI, diffuse axonal injury; GMS, Glasgow Motor Score.

$\mathrm{CPP}$, cerebral perfusion pressure.

microhemorrhages than $\mathrm{T} 2 * \mathrm{GRE}^{26}$ SWI has previously been demonstrated to show significantly more DAI-associated microhemorrhages than $\mathrm{T} 2 * \mathrm{GRE},{ }^{27}$ findings supported by the present study. SWI sequences are fast to acquire and may allow depiction of hemorrhagic lesions superior to T2*GRE. ${ }^{28,29}$ The SWI sequence may be more difficult to interpret than T2*GRE and DWI sequences, because deoxygenated blood in veins can be misinterpreted as hemorrhagic lesions.

The evaluation of inter-rater agreement shows, however, similar high values for all three sequences, illustrating the robustness of the SWI technique even for less experienced raters. SWI also identified a significantly higher number of lesions than the DW sequence; direct comparison between these two sequences is misleading, however, because they in part visualize different lesion types.

In this study, we analyzed DW images, which are sensitized to motion on a molecular level, but we did not use apparent diffusion coefficient (ADC) maps in which diffusion has been calculated and visualized. On DW images, diffusion reduction caused by intracellular edema increases the signal intensity, but the signal intensity may also be increased by high T2 signal.

Diffusion tensor imaging (DTI), which measures the degree of anisotropy of water diffusion in white matter fibers, is a more sensitive measure for DAI-associated lesions. ${ }^{30}$ Also, depicted lesions on DTI seem to be stable from the acute period up to 5 years post-injury. ${ }^{31}$ Assessment of DAI related changes with DTI, however, requires extensive post-processing techniques and statistical analysis, whereas this is not needed for the sequences used in this study. In addition, the sequences used in this study have the advantage of being widely available and well familiar to neuroradiologists.

\section{The timing of $M R I$}

Both nonhemorrhagic and hemorrhagic DAI lesions appear less conspicuous over time, ${ }^{17,18}$ although residues of microhemorrhagic lesions may persist for months to years. ${ }^{10,32}$ In this study, patients were examined with MRI within the first week post-injury, allowing improved anatomical grading and quantification of anatomical distribution for prediction of outcome. Although hemorrhagic lesions seem to persist beyond the acute period postinjury, ${ }^{17,33}$ the timing of the MRI seems to be of particular im- portance when evaluating DWI sequences. Studies evaluating the evolution of DWI lesions within the first month of trauma in severe TBI are lacking.

In stroke patients, however, the ADC of lesion has been found to decrease until the fourth day, then gradually increase during the first week post-stroke to pseudonormal during the second week and to supranormal thereafter. ${ }^{34}$ Therefore lesions may be isointense to brain parenchyma and not depicted on DWI if the MRI is performed beyond the first week post-injury. The optimal timing for MRI after TBI for predicting outcome needs to be further elucidated in future studies, but likely, it should preferably be performed within the first week post-injury for reliable prognostication.

\section{Anatomical localization and long-term outcome}

This study included an anatomical localization of DAIassociated lesions. Lesions were commonly found in the frontal and temporal lobes of the cerebral hemispheres as well as in the body and splenium of the corpus callosum. Brainstem lesions were seen in a high proportion of patients, mainly in the mesencephalon and the upper pons. In addition, lesions in central supratentorial areas as the thalamus, basal ganglia, and internal capsule were frequently identified, particularly on SWI sequences.

The number of lesions in the cerebral hemispheres, cerebellum, and corpus callosum did not show any significant association to long-term outcome. In a study by Moen and colleagues ${ }^{35}$ that included 128 patients with TBI examined with MRI within 0-28 days, DWI lesions in the corpus callosum were the most important predictive MRI variable in contradiction to the results in this report. That study, however, included a heterogeneous cohort of moderate and severe TBI, with evacuated mass lesions in $31 \%$ of patients with severe TBI. Direct comparison with this study is

Table 7. Extended Magnetic Resonance Imaging

Classification System for Diffuse Axonal InJury

and Distribution of Patient Outcomes in Different

Stages of Diffuse Axonal Injury Measured By Glasgow Outcome Scale Extended after $>6$ MONThS

\begin{tabular}{|c|c|c|c|}
\hline & $\begin{array}{l}\text { Age } \\
<30\end{array}$ & $\begin{array}{l}\text { Age } \\
\geq 30\end{array}$ & GOSE \\
\hline $\begin{array}{l}\text { Stage I } \\
\text { Hemispheric } \\
\text { lesions }\end{array}$ & Ia & $\mathrm{Ib}$ & $\begin{array}{l}\text { Ia: No cases } \\
\quad \text { Ib: GOSE 5-6 }(n=2)\end{array}$ \\
\hline $\begin{array}{l}\text { Stage II } \\
\text { Corpus callosum } \\
\text { lesions }\end{array}$ & IIa & IIb & $\begin{array}{c}\text { IIa: GOSE 7-8 }(n=2) \\
\text { GOSE 5-6 }(n=2) \\
\text { IIb: GOSE 5-6 }(n=1) \\
\text { GOSE 3-4 }(n=1)\end{array}$ \\
\hline $\begin{array}{l}\text { Stage III } \\
\quad \text { Brainstem lesions }{ }^{\dagger}\end{array}$ & IIIa & IIIb & $\begin{array}{c}\text { IIIa: GOSE 7-8 }(n=5) \\
\text { GOSE 5-6 }(n=1) \\
\text { GOSE 3-4 }(n=1) \\
\text { IIIb: GOSE 7-8 }(n=1) \\
\text { GOSE 5-6 }(n=2) \\
\text { GOSE 3-4 }(n=1)\end{array}$ \\
\hline $\begin{array}{l}\text { Stage IV } \\
\text { Lesions in SN or } \\
\text { mesencephalic } \\
\text { tegmentum }\end{array}$ & IVa & $\mathrm{IVb}$ & $\begin{array}{c}\text { IVa: GOSE 7-8 }(n=1) \\
\text { GOSE 5-6 }(n=2) \\
\text { GOSE 3-4 }(n=2) \\
\text { IVb: GOSE 5-6 }(n=1) \\
\text { GOSE 3-4 }(n=3) \\
\text { GOSE 1-2 }(n=1)\end{array}$ \\
\hline
\end{tabular}

†Substantia nigra and mesencephalic tegmentum excepted. GOSE, Glasgow Outcome Scale extended; SN, substantia nigra. 
therefore difficult. Radiological methods were also different. They used ADC maps to see diffusion abnormalities and T2-weighted fluid attenuated inversion recovery images to see other lesions. SWI sequences were not included, which may explain the lower number of identified brainstem lesions.

Deep supratentorial lesions in basal ganglia, internal capsule, and especially in the thalamus have been proposed to predict poor outcomes. ${ }^{35}$ Thalamic lesions have also been suggested as a primary feature of the vegetative state. ${ }^{4,36}$ This association with longterm outcome could be seen in deep supratentorial lesions with DW sequences, but the association lost significance after adjustment for other prognostic factors in our study. DAI-associated lesions in the mesencephalon identified on SWI sequences had a strong association with poor long-term outcome in univariate analysis. After adjustment for other prognostic factors, however, only DAIassociated lesions in the region of the substantia nigra and the tegmentum of the mesencephalon showed an independent prognostic value.

The role of brainstem lesions in DAI and in severe TBI in general has been discussed in multiple studies. ${ }^{3,37-39}$ It is debated whether the different anatomical localizations are the result of distinct injury mechanisms. Superficial brainstem lesions may be the effect of brainstem impact at the tentorial edge, whereas deep located lesions may represent a true traumatic axonal injury mechanism and therefore have a stronger relation to poor long-term outcome. ${ }^{40}$

The general value of the location of hemorrhagic lesions in the brainstem after DAI for prognosis is still uncertain, but deep posterior and bilateral lesions have been proposed to be particularly related to poor outcomes. ${ }^{3,40,41}$ No previous study, however, has included a clear definition of lesion location in the mesencephalon and upper pons where traumatic axonal injuries were predominantly found. In our study, the mesencephalon was anatomically divided into three regions: (1) crus cerebri, (2) substantia nigra and mesencephalic tegmentum, and (3) mesencephalic tectum. Lesions in crus cerebri and the tectum represented a more superficial location and did not show significant association with poor long-term outcomes in the multivariate analysis. More deeply located lesions in the region corresponding to the substantia nigra and the mesencephalic tegmentum, however, had an independent prognostic value. Further, previous studies are mainly based on lesions seen with $\mathrm{T} 2 * \mathrm{GRE}, 3,35,37-39$ and, to our knowledge, this is the first study to evaluate the role of anatomical location of brainstem lesions on SWI in DAI.

With the increased sensitivity for detection of hemorrhagic lesions compared with $\mathrm{T} 2 * \mathrm{GRE}$, SWI gives the possibility of convenient anatomical localization of brainstem lesions. It is possible
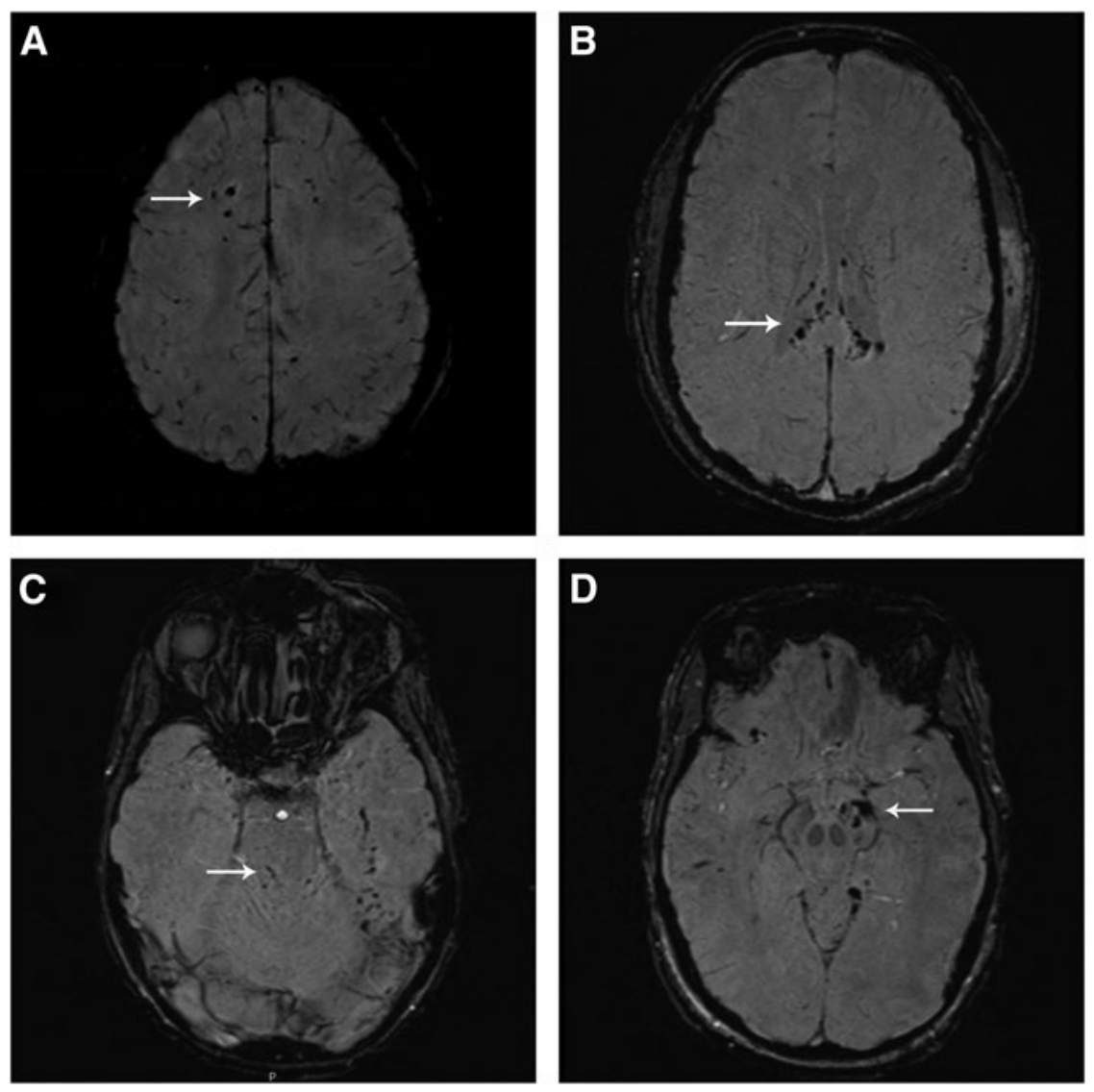

FIG. 2. (A) Diffuse axonal injury (DAI) stage I: susceptibility-weighted imaging (SWI) of a 19-year-old male involved in a motor vehicle accident. The image reveals hemorrhagic lesions in the supratentorial white matter. Patient had a good recovery (Glasgow Outcome Scale extended [GOSE] 7) $>6$ months after trauma. (B) DAI stage II: SWI image of a 23 -year-old male involved in a motor vehicle accident. The image reveals hemorrhagic lesions in the splenium and body of the corpus callosum. The patient was moderately disabled (GOSE 6) >6 months after trauma. (C) DAI stage III: SWI image of an 18-year-old male involved in a motor vehicle accident. The image reveals hemorrhagic lesions in the dorsal part of the upper pons. The patient was moderately disabled (GOSE 5) $>6$ months after trauma. (D) DAI stage IV: SWI image of a 35-year-old female involved in a horseback riding accident. The image reveals hemorrhagic lesions in the left crus cerebri and in the substantia nigra. The patient was severely disabled (GOSE 3) $>6$ months after trauma. 
that lesions in the central region of the mesencephalon may be missed when only $\mathrm{T} 2 * \mathrm{GRE}$ is used, and this study concludes that they have strong association with poor outcome.

\section{Anatomical DAl grading}

In this study, the widely used histopathological grading system of Adams and coworkers ${ }^{7}$ introduced in 1989 was applied to modern MRI imaging. In this patient cohort with severe TBI and DAI, 70\% of patients showed hemorrhagic or nonhemorrhagic lesions in the brainstem consistent with DAI grade 3 . This is slightly higher than in previous studies reporting brainstem injuries in approximately half of patients with severe $\mathrm{TBI},{ }^{3,42}$ likely explained by the inclusion of SWI sequences yielding a higher sensitivity for hemorrhagic brainstem lesions.

DAI grading according to Adams and coworkers, ${ }^{7}$ however, did not show significant relation to long-term outcome. Thus, this DAI grading alone seems to have unreliable prognostic significance in patients with severe DAI who present with deep unconsciousness, in which lesions in deeper locations including the brainstem seem to be a common finding. Further subdivision of DAI-associated brainstem lesions is therefore warranted for predicting outcomes. This study identified the presence of lesions in the substantia nigra and the tegmentum of the mesencephalon as a promising indicator of poor long-term outcome. The presence or absence of these lesions can be used to complement the DAI grading system of Adams and associates. ${ }^{7}$

\section{Prognostic impact of clinical factors}

Age is a well-studied prognostic factor in TBI, where multiple studies have shown higher mortality and poorer long-term outcomes in older patients. Age adds an increasing probability of poor long-term outcome in a continuous fashion. ${ }^{43-45}$ In this study, mean age of patients was 31.2 years (range 16-60), thus a younger than the general population in severe TBI. ${ }^{46-48}$ Age was a strong independent prognostic factor of poor long-term outcome after adjustment for other prognostic factors, both as a continuous variable and as a dichotomized category of age below and above 30 years. These findings may indicate a decrease in brain plasticity and resilience to severe traumatic axonal injury with increasing age.

An association between poor long-term outcome and decreased level of consciousness measured with GMS at admission and discharge was seen in univariate analysis. The study did not show any independent prognostic predictive value of GMS after adjustment for other factors, however. Generally, DAI is acquired after a high energy motor vehicle accident, where injuries to multiple organ systems are not uncommon. This is emphasized by the relatively high mean Injury Severity Score ${ }^{49}$ (24.3, range 16-45) and number of patients with extracranial injury ( 22 of 30 patients) in this patient cohort, which complicates the GCS evaluation.

\section{Extended anatomical MRI classification}

A number of MRI-based classification systems have been proposed in severe TBI. In the classification system of Firsching and colleagues, ${ }^{42}$ brainstem injuries were classified according to their rostrocaudal and unilateral or bilateral extent in the brainstem, independent of degree of supratentorial injuries. A more recent study added a mechanism-based approach to this classification scheme, including mechanism of brainstem injury to the classification. ${ }^{50}$ Whereas these classification systems included patients with brainstem injuries after both focal and diffuse TBI, our system is focused on DAI.
To our knowledge, there is currently no other classification system for DAI differentiating anatomical lesion location in the brainstem. The Adams classification system, ${ }^{7}$ the most widely used classification for DAI, seems to lack prognostic value in patients with DAI who present with deep unconsciousness. Therefore, a new classification system is needed for better prediction of outcome in these patients.

From the results of this study, we propose an extended classification system for DAI based on the DAI grading system of Adams and associates ${ }^{7}$ that is simple to use and adds significant prognostic value. Age $\geq 30$ years and lesions in the substantia nigra or mesencephalic tegmentum were included in the MRI-based classification, and DAI was classified in four stages with age-based subdivision (Table 7 and Fig. 2A-D). This classification needs further validation, and we are currently including additional patients with severe TBI and DAI to validate the classification system.

In addition, the classification system could be confounded by a number of study limitations. First, the number of patients is small, and the three MRI sequences included in the evaluation were not available in all patients, which may limit the conclusions that can be drawn by the study. Further, it is possible that the findings may have been biased by patient selection, because a number of patients with suspected DAI by clinical and CT criteria could not be examined with MRI early. The independent association with poor long-term outcome of age $\geq 30$ and lesions in the region of the substantia nigra and mesencephalic tegmentum found in this patient material, however, justifies preliminary use of this classification system for predicting patient outcome.

\section{Conclusions}

In this study, 30 patients with severe TBI and DAI were evaluated with MRI within 1 week after trauma. The SWI sequence was superior to the T2*GRE and DW sequences in identifying DAIassociated lesions in the early phase after severe DAI. Lesions in the brainstem in conjunction with hemispheric and corpus callosum lesions were identified in a large proportion of patients. The DAI grading using the classification system proposed by Adams and coworkers, ${ }^{7}$ however, did not correlate significantly with outcome.

Only advanced age and brainstem lesions anatomically localized in the substantia nigra and the tegmentum of the mesencephalon identified by SWI sequences had an independent prognostic value for predicting poor long-term outcome after adjustment for other factors. Based on the findings from this study, we propose a new extended anatomical MRI classification system in patients with DAI for early prediction of outcome.

\section{Author Disclosure Statement}

No competing financial interests exist.

\section{References}

1. Coronado, V.G., Xu, L., Basavaraju, S.V., McGuire, L.C., Wald, M.M., Faul, M.D., Guzman, B.R., and Hemphill, J.D. (2011). Surveillance for traumatic brain injury-related deaths-United States, 1997-2007. MMWR Surveill. Summ. 60, 1-32.

2. Gentry, L.R. (1994). Imaging of closed head injury. Radiology 191, $1-17$.

3. Skandsen, T., Kvistad, K.A., Solheim, O., Strand, I.H., Folvik, M., and Vik, A. (2010). Prevalence and impact of diffuse axonal injury in patients with moderate and severe head injury: a cohort study of early magnetic resonance imaging findings and 1-year outcome. J. Neurosurg. 113, 556-563.

4. Kampfl, A., Franz, G., Aichner, F., Pfausler, B., Haring, H.P., Felber, S., Luz, G., Schocke, M., and Schmutzhard, E. (1998). The persistent 
vegetative state after closed head injury: clinical and magnetic resonance imaging findings in 42 patients. J. Neurosurg. 88, 809-816.

5. Graham, D.I., Adams, J.H., Murray, L.S., and Jennett, B. (2005). Neuropathology of the vegetative state after head injury. Neuropsychol. Rehabil. 15, 198-213.

6. Adams, J.H., Jennett, B., Murray, L.S., Teasdale, G.M., Gennarelli, T.A., and Graham, D.I. (2011). Neuropathological findings in disabled survivors of a head injury. J. Neurotrauma 28, 701-709.

7. Adams, J.H., Doyle, D., Ford, I., Gennarelli, T.A., Graham, D.I., and Mclellan, D.R. (1989). Diffuse axonal injury in head injury: definition, diagnosis and grading. Histopathology 15, 49-59.

8. Marshall, L.F., Marshall, S.B., Klauber, M.R., Clark, M.B., Eisenberg, H.M., Jane, J.A., Luerssen, T.G., Marmarou, A., and Foulkes, M.A. (1991). A new classification of head injury based on computerized tomography. J. Neurosurg. 75, S14-S20.

9. Gennarelli, T.A., Thibault, L.E., Adams, J.H., Graham, D.I., Thompson, C.J., and Marcincin, R.P. (1982). Diffuse axonal injury and traumatic coma in the primate. Ann. Neurol. 12, 564-574.

10. Ripoll, M., Siosteen, B., Hartman, M., and Raininko, R. (2003). MR detectability and appearance of small experimental intracranial hematomas at $1.5 \mathrm{~T}$ and $0.5 \mathrm{~T}$. A 6-7-month follow-up study. Acta Radiol. 44, 199-205.

11. Scheid, R. Preul, C., Gruber, O., Wiggins, C., and von Cramon, D.Y. (2003). Diffuse axonal injury associated with chronic traumatic brain injury: evidence from T2*-weighted gradient-echo imaging at $3 \mathrm{~T}$. AJNR Am. J. Neuroradiol. 24, 1049-1056.

12. Xu, J., Rasmussen, I.A., Lagopoulos, J., and Håberg, A. (2007). Diffuse axonal injury in severe traumatic brain injury visualized using high-resolution diffusion tensor imaging. J. Neurotrauma 24, 753-765.

13. Li, X., and Feng, D. (2009). Diffuse axonal injury: novel insights into detection and treatment. J. Clin. Neurosci. 16, 614-619.

14. Chastain, C.A., Oyoyo, U.E., Zipperman, M., Joo, E., Ashwal, S., Shutter, L.A., and Tong, K.A. (2009). Predicting outcomes of traumatic brain injury by imaging modality and injury distribution. J. Neurotrauma 26, 1183-1196.

15. Yanagawa, Y., Sakamoto, T., Takasu, A., and Okada, Y. (2009). Relationship between maximum intracranial pressure and traumatic lesions detected by $\mathrm{T} 2 *$-weighted imaging in diffuse axonal injury. $\mathrm{J}$. Trauma 66, 162-165.

16. Chelly, H., Chaari, A., Daoud, E., Dammak, H., Medhioub, F., Mnif, J., Hamida, C.B., Bahloul, M., and Bouaziz, M. (2011). Diffuse axonal injury in patients with head injuries: an epidemiologic and prognosis study of 124 cases. J. Trauma 71, 838-846.

17. Moen, K.G., Skandsen, T., Folvik, M., Brezova, V., Kvistad, K.A., Rydland, J., Manley, G.T., and Vik, A. (2012). A longitudinal MRI study of traumatic axonal injury in patients with moderate and severe traumatic brain injury. J. Neurol. Neurosurg. Psychiatry 83, 11931200 .

18. Messori, A., Polonara, G., Mabiglia, C., and Salvolini, U. (2003). Is haemosiderin visible indefinitely on gradient-echo MRI following traumatic intracerebral haemorrhage? Neuroradiology 45, 881886 .

19. Elf, K., Nilsson, P., and Enblad, P. (2002). Outcome after traumatic brain injury improved by an organized secondary insult program and standardized neurointensive care. Crit. Care Med. 30, 21292134.

20. Skoglund, K., Enblad, P., Hillered, L., and Marklund, N. (2012). The neurological wake-up test increases stress hormone levels in patients with severe traumatic brain injury. Crit. Care Med. 40, 216-222.

21. Howells, T.P., Jones, P.A., Piper, I.R., Souter, M.J., and Miller, J.D. (1995). Design of a research database for the study of secondary insults following head injury [abstract]. J. Neurotrauma 12:471.

22. Czosnyka, M., Smielewski, P., Kirkpatrick, P., Laing, R., Menon, D., and Pickard, J.D. (1997). Continuous assessment of the cerebral vasomotor reactivity in head injury. Neurosurgery 41, 11-19.

23. Howells, T., Lewén, A., Sköld, M.K., Ronne-Engström, E., and Enblad, P. (2012). An evaluation of three measures of intracranial compliance in traumatic brain injury patients. Intensive Care Med. 38, 1061-1068.

24. Wilson, J.T., Pettigrew, L.E., and Teasdale, G.M. (1998). Structured interviews for the Glasgow Outcome Scale and the extended Glasgow Outcome Scale: guidelines for their use. J. Neurotrauma 15, 573-585.
25. Strich, S.J. (1956). Diffuse degeneration of the cerebral white matter in severe dementia following head injury. J. Neurol. Neurosurg. Psychiatry 19, 163-185.

26. Haacke, E.M., Mittal, S., Wu, Z., Neelavalli, J., and Cheng, Y.C. (2008). Susceptibility-weighted imaging: technical aspects and clinical applications, part 1. AJNR Am. J. Neuroradiol. 30, 19-30.

27. Tong, K.A., Ashwal, S., Holshouser, B.A., Shutter, L.A., Herigault, G., Haacke, E.M., and Kido, D.K. (2003). Hemorrhagic shearing lesions in children and adolescents with posttraumatic diffuse axonal injury: improved detection and initial results. Radiology 227, 332339.

28. Tong, K.A., Ashwal, S., Holshouser, B.A., Nickerson, J.P., Wall, C.J., Shutter, L.A., Osterdock, R.J., Haacke, E.M., and Kido, D. (2004). Diffuse axonal injury in children: clinical correlation with hemorrhagic lesions. Ann. Neurol. 56, 36-50.

29. Mittal, S., Wu, Z., Neelavalli, J., and Haacke, E.M. (2009). Susceptibility-weighted imaging: technical aspects and clinical applications, part 2. AJNR Am J Neuroradiol. 30, 232-252.

30. Inglese, M., Makani, S., Johnson, G., Cohen, B.A., Silver, J.A., Gonen, O., and Grossman, R.I. (2005). Diffuse axonal injury in mild traumatic brain injury: a diffusion tensor imaging study. J. Neurosurg. 103, 298-303.

31. Dinkel J, Drier A, Khalilzadeh O, Perlbarg V, Czernecki V, Gupta R, Gomas F, Sanchez P, Dormont D, Galanaud D, Stevens RD, and Puybasset L; for NICER (Neuro Imaging for Coma Emergence and Recovery) Consortium. (2014). Long-term white matter changes after severe traumatic brain injury: a 5-year prospective cohort. AJNR Am. J. Neuroradiol. 35, 23-29.

32. Wardlaw, J.M., and Statham, P.F. (2000). How often is haemosiderin not visible on routine MRI following traumatic intracerebral haemorrhage? Neuroradiology 42, 81-84.

33. Moen, K., Vik, A., Olsen, A., Skandsen, T., Håberg, A.K., Evensen, K.A., and Eikenes, L. (2016). Traumatic axonal injury: relationships between lesions in the early phase and diffusion tensor imaging parameters in the chronic phase of traumatic brain injury. J. Neurosci. Res. 94, 623-635.

34. Lansberg, M.G., Thijs, V.N., O’Brien, M.W., Ali, J.O., de Crespigny, A.J., Tong, D.C., Moseley, M.E., and Albers, G.W. (2001) Evolution of apparent diffusion coefficient, diffusion-weighted, and T2-weighted signal intensity of acute stroke. AJNR Am. J. Neuroradiol. 22, 637644.

35. Moen, K.G., Brezova, V., Skandsen, T., Håberg, A.K., Folvik, M., and Vik, A. (2014). Traumatic axonal injury: the prognostic value of lesion load in corpus callosum, brain stem, and thalamus in different magnetic resonance imaging sequences. J Neurotrauma 31, 14861496.

36. Adams, J.H., Graham, D.I., and Jennet, B. (2000). The neuropathology of the vegetative state after an acute brain insult. Brain 123, 13271338 .

37. Firsching, R., Woischneck, D., Diedrich, M., Klein, S., Rückert, A., Wittig, H., and Döhring, W. (1998). Early magnetic resonance imaging of brainstem lesions after severe head injury. J. Neurosurg. 89, 707-712.

38. Firsching, R., Woischneck, D., Klein, S., Ludwig, K., and Döhring, W. (2002). Brain stem lesions after head injury. Neurol. Res. 24, 145146.

39. Chew, B.G., Spearman, C.M., Quigley, M.T., and Wilberger, J.E. (2012). The prognostic significance of traumatic brainstem injury detected on T2-weighted MRI. J. Neurosurg. 117, 722-728.

40. Shibata, Y., Matsumura, A., Meguro, K., and Narushima, K. (2000). Differentiation of mechanism and prognosis of traumatic brain stem lesions detected by magnetic resonance imaging in the acute stage. Clin. Neurol. Neurosurg. 102, 124-128.

41. Hilario, A., Ramos, A., Millan, J.M., Salvador, E., Gomez, P.A., Cicuendez, M., Diez-Lobato, R., and Lagares, A. (2012). Severe traumatic head injury: prognostic value of brain stem injuries detected at MRI. AJNR Am. J. Neuroradiol. 33, 1925-1931.

42. Firsching, R., Woischneck, D., Klein, S., Reissberg, S., Döhring, W., and Peters, B. (2001). Classification of severe head injury based on magnetic resonance imaging. Acta Neurochir. (Wien) 143, $263-$ 271.

43. The Brain Trauma Foundation. (2000). The American Association of Neurological Surgeons. The Joint Section on Neurotrauma and Critical Care. Age. J. Neurotrauma 17, 573-581. 
44. Mushkudiani, N.A., Engel, D.C., Steyerberg, E.W., Butcher, I., Lu, J., Marmarou, A., Slieker, F., McHugh, G.S., Murray, G.D., and Maas, A.I. (2007). Prognostic value of demographic characteristics in traumatic brain injury: results from the IMPACT study. J. Neurotrauma 24, 259-269.

45. Roozenbeek, B., Maas, A.I., and Menon, D.K. (2013). Changing patterns in the epidemiology of traumatic brain injury. Nat. Rev. Neurol. 9, 231-236.

46. Rusnak, M., Janciak, I., Majdan, M., Wilbacher, I., and Mauritz, W. (2007). Severe traumatic brain injury in Austria I: introduction to the study. Wien klin. Wochenschr. 119, 23-28.

47. Andriessen, T.M., Horn, J., Franschman, G., van der Naalt, J., Haitsma, I., Jacobs, B., Steyerberg, E.W., and Vos, P.E. (2011) Epidemiology, severity classification, and outcome of moderate and severe traumatic brain injury: a prospective multicenter study. J. Neurotrauma 28, 2019-2031.

48. Stocchetti, N., Paternó, R., Citerio, G., Beretta, L., and Colombo, A. (2012). Traumatic brain injury in an aging population. J. Neurotrauma 29, 1119-1125.
49. Baker, S.P., O’Neill, B., Haddon, W., Jr, and Long, W.B (1974). The injury severity score: a method for describing patients with multiple injuries and evaluating emergency care. J. Trauma 14, 187-196.

50. Mannion, R.J., Cross, J., Bradley, P., Coles, J.P., Chatfield, D., Carpenter, A., Pickard, J.D., Menon, D.K., and Hutchinson, P.J. (2007). Mechanism-based MRI classification of traumatic brainstem injury and its relationship to outcome. J. Neurotrauma 24, 128-135.

Address correspondence to:

Sami Abu Hamdeh, MD

Department of Neuroscience, Neurosurgery

Uppsala University Hospital

75185 Uppsala

Sweden

E-mail: sami.abu.hamdeh@neuro.uu.se 